\title{
Psychoonkologie: Möglichkeiten und Grenzen psychosozialer Hilfen bei einer Krebserkrankung
}

\section{Psycho-oncology: Possibilities and Limitations of Psychosocial Help in Cancer}

\author{
Autoren \\ Kim Alisha Drommelschmidt' ${ }^{1}$, André Karger² \\ Institute \\ 1 Klinik für Onkologische Rehabilitation in der Klinik für \\ Tumorbiologie, Universitätsklinikum Freiburg \\ 2 Klinisches Institut für Psychosomatische Medizin und \\ Psychotherapie, Universitätsklinikum Düsseldorf
}

\author{
Schlüsselwörter \\ Psychoonkologie, sektorübergreifend, flächendeckende \\ Versorgung
}

Key words

Psycho-oncology, trans-sectoral, comprehensive cancer care

\author{
Bibliografie \\ DOI https://doi.org/10.1055/a-0638-9921 \\ Deutsche Zeitschrift für Onkologie 2018; 50: 168-171 \\ (c) Georg Thieme Verlag KG Stuttgart · New York \\ ISSN 1617-5891
}

Korrespondenzadresse

Kim Alisha Drommelschmidt

Klinik für Onkologische Rehabilitation in der Klinik für

Tumorbiologie

UKF Reha gGmbH

Breisacher Straße117

79106 Freiburg

kim.alisha.drommelschmidt@ukf-reha.de

\section{ZUSAMENFASSUNG}

Die Psychoonkologie ist mittlerweile ein fester Bestandteil der multiprofessionellen onkologischen Behandlung und kann Patienten und ihre Angehörigen bei der Krankheitsbewältigung und im Umgang mit psychosozialen Problemen unterstützen. Die Effektivität von psychoonkologischen Interventionen ist vielseitig belegt, jedoch gibt es hinsichtlich der Sicherstellung einer sektorübergreifenden Kommunikation zwischen den Behandlern sowie einer flächendeckend verfügbaren, bedarfsgerechten psychosozialen Versorgung noch Verbesserungsbedarf.

\section{ABSTRACT}

Psycho-oncology is part of the multi-professional cancer care and helps patients and their relatives in coping with cancer and psychosocial problems. The effectiveness of psychooncological interventions is well documented. Trans-sectoral communication (continuity of care) between the healthcare providers and comprehensive available adequate psychosocial care should be improved in the next years.

\section{Bedarf}

Mehr als ein Drittel der Patienten* mit einer Krebserkrankung leidet unter Ängsten oder Depressionen. Nach aktueller Studienlage benötigt jedoch deutlich mehr als ein Drittel der Krebspatienten eine begleitende psychoonkologische Therapie. Die häufigsten komorbiden psychischen Erkrankungen onkologischer Patienten sind

- Anpassungsstörungen (Punktprävalenz 8.0\%),

- affektive Störungen (Punktprävalenz 10,8\%) und

- Angststörungen (Punktprävalenz 13,5\%) [1].

* Es wird im Manuskript auf die gleichzeitige Verwendung männlicher und weiblicher Sprachformen verzichtet.
Weit größer noch ist der Anteil der Patienten, die eine erhöhte Belastung (aber keine psychische Störung) aufweisen. Dabei können sich die Belastungsfaktoren bei Betroffenen auf einem Kontinuum von normalen Ängsten, Sorgen und Traurigkeit sowie subsyndromalen Zuständen erstrecken. Hier erleben die Betroffenen deutliche Problem- und Bedürfnislagen, die mit einer starken emotionalen Belastung und vereinzelten psychischen Symptomen verbunden sind, jedoch nicht die Kriterien einer psychischen Erkrankung (gemäß ICD-10) erfüllen. Vulnerabilitätsfaktoren für die Entwicklung einer psychischen Erkrankung sind eine erhöhte somatische Symptombelastung, Schmerzen, Fatigue und eine bereits in der Vorgeschichte bestehende psychische Erkrankung. 
Patienten, die neben ihrer Krebserkrankung auch unter einer psychischen Störung leiden, sind nicht nur in ihrer Lebensqualität eingeschränkt. Die psychische Erkrankung wirkt sich negativ auf den Verlauf der Krebserkrankung aus und führt zu einer erhöhten Morbidität (bspw. mehr Nebenwirkungen während der Therapie) und Mortalität [2].

\section{Was sind typische psychosoziale Herausforderungen bei Krebs?}

Typische Herausforderungen für Krebspatienten sind neben dem Umgang mit Ängsten vor Tod und Sterben und dem Verlust der Autonomie, die Belastung durch Schmerzen oder körperliche Beeinträchtigungen (z. B. Anlage eines künstlichen Darmausgangs bei Darmkrebs), existentielle und spirituelle Fragen (z. B. nach dem Sinn des Lebens und der Erkrankung), aber auch soziale Beeinträchtigungen, beispielsweise durch längere Arbeitsunfähigkeit und eingeschränkte soziale Kontakte oder partnerschaftliche und familiäre Konflikte aufgrund eines veränderteren Rollengefüges.

\section{Merke}

Krebs betrifft als Wir-Erkrankung immer die ganze Familie und psychosoziale Probleme bestehen oft nach Abschluss der akuten onkologischen Therapie noch über Jahre hinweg.

\section{Was ist Psychoonkologie?}

Die Psychoonkologie, synonym auch als Psychosoziale Onkologie bezeichnet, ist heutzutage ein unverzichtbarer Bestandteil der multiprofessionellen onkologischen Behandlungen.

Als interdisziplinäres Fachgebiet beschäftigt sich die Psychoonkologie mit dem Erleben und Verhalten sowie den Ressourcen von Krebserkrankten und ihren psycho-sozialen Problemen im Zusammenhang mit der Diagnose, Behandlung und dem Verlauf der Krebserkrankung. Die Psychoonkologie kann Betroffene bei individuellen, familiären und sozialen Prozessen der Krankheitsverarbeitung und bei dem Umgang mit Krankheitsfolgen unterstützen. Dabei schließt die Hilfe auch Angehörige und das soziale Umfeld in die Beratung und/oder Behandlung mit ein.

Die dazugehörige psychoonkologische Forschung konzentriert sich auf die Bedeutung psychologischer und sozialer Faktoren für die Entstehung, Früherkennung, Diagnostik, Behandlung, Rehabilitation, Nachsorge sowie den gesamten Verlauf einer Tumorerkrankung. Ziel ist es, deren Wechselwirkungen wissenschaftlich zu untersuchen, um die gewonnenen Erkenntnisse in der Prävention, Früherkennung, Diagnostik, Behandlung, Rehabilitation, ambulanten Versorgung und Palliativbetreuung von Betroffenen nutzbar zu machen.

Beispielsweise konnte die Forschung zeigen, dass zwar frühkindliche Stresserfahrungen das Risiko, später im Leben an Krebs zu erkranken erhöhen. Die Annahme einer spezifischen Persönlichkeitsstruktur, der sog. Krebspersönlichkeit, die das Auftreten von Krebs begünstigen soll, gilt inzwischen jedoch als widerlegt [3].

\section{S3-Leitlinie „Psychoonkologische Diagnostik, Beratung und Behandlung von erwachsenen Krebspatienten“}

Ein Meilenstein für die Bedeutsamkeit und die jüngsten positiven Entwicklungen in der psychoonkologischen Versorgung war die 2014 erschienene, für alle Tumorerkrankungen übergreifende S3Leitlinie „Psychoonkologische Diagnostik, Beratung und Behandlung von erwachsenen Krebspatienten“. Diese definiert evidenzbasiert psycho-soziale Versorgungsstandards bei verschiedenen Krebserkrankungen und ist aktuell auch eine wichtige Grundlage für die Zertifizierungskriterien von Organzentren (C, auf ein Organ spezialisiert), onkologischen Zentren (CC, Behandlung mehrerer Tumorarten) und onkologischen Spitzenzentren (CCC, Schwerpunkt auf der Entwicklung neuer Therapiestrategien). Damit alle Zentren trotz ihrer unterschiedlichen Aufgabenspektren einheitliche Leistungsstandards erfüllen, unterziehen sich diese freiwillig strengen Qualitätskontrollen. Deren Anforderungskatalog wurde 2013 von der Deutschen Krebsgesellschaft und der Deutschen Krebshilfe harmonisiert und in einem einheitlichen Erhebungsbogen zusammengefasst. Seitdem ist er Bestandteil des Nationalen Zertifizierungsprogramms Krebs.

\section{Merke}

Die Inhalte der S3- Leitlinie verdeutlichen zudem die Bedeutung eines multizentrischen, interdisziplinären und patientenorientierten Behandlungsansatzes in der Onkologie.

Ziel ist die Zusammenarbeit aller an der Behandlung beteiligten Berufsgruppen, wie Ärzten, Psychologen, Sozialarbeitern, Pflegekräften, Kunsttherapeuten, Ergotherapeuten und Seelsorgern, um dem Patienten eine Behandlung zu ermöglichen, die konsequent auf seine individuellen Bedürfnisse abgestimmt ist [1].

Eine weitere Zielsetzung ist, dass diese Zusammenarbeit sektorübergreifend alle Professionen und Institutionen der psychoonkologischen Versorgung miteinbezieht: die Akutkrankenhäuser, Rehakliniken sowie die ambulanten Behandler, u. a. Ärzte in der Nachsorge, Psychotherapeuten und Krebsberatungsstellen [4].

Die Festlegung einheitlicher psychoonkologischer Versorgungsstandards führte in den letzten Jahren bereits zu einem Ausbau von psychoonkologischen Konsiliar- und Liaisondiensten in Krankenhäusern sowie zur Förderung ambulanter Angebote wie Krebsberatungsstellen und steigerte darüber hinaus das Interesse für neue Forschungsansätze [5].

Allerdings sind die beschriebenen standardisierten, interprofessionellen Behandlungskonzepte in der Onkologie in Deutschland insgesamt kaum flächendeckend umgesetzt. Als problematisch erweisen sich v. a. die Organisation der Kommunikation zwischen den einzelnen Professionen, der konsequente Einbezug der Patientenperspektive sowie die sektorenübergreifende Koordination der Behandlung. An den Unikliniken Düsseldorf und Aachen gibt es ein integriertes Versorgungskonzept Psychoonkologie in Kooperation mit der AOK Rheinland/Hamburg und niedergelassenen Psychotherapeuten, bei dem durch eine Gesundheitslotsin die sektorübergreifende Versorgung und Vernetzung der verschiedenen beteiligten Fachdisziplinen und Institutionen des Gesundheitswesens koordiniert werden [6]. Leider sind dies jedoch bislang noch Einzelfälle. 


\section{Wie können psychosoziale Belastungen erkannt werden?}

Für eine zielgerichtete und effiziente psychoonkologische Versorgung sind standardisierte Vorgehensweisen zur frühzeitigen Erkennung von unterstützungsbedürftigen Patienten und deren individuellem Behandlungsbedarf besonders wichtig. Es ist nicht immer ohne weiteres erkennbar, welche Patienten im Einzelnen Unterstützung benötigen [7]. Beispielsweise sind viele Patienten vordringlich mit der Organisation und Bewältigung ihrer Krebsbehandlung beschäftigt und achten dabei weniger auf ihre psychische Belastung. Auch wünscht nicht jeder psychisch belastete Patient unbedingt psychosoziale Unterstützung.

\section{Merke}

Ein Lösungsansatz dieser Problematik ist ein einheitliches Screening für alle onkologischen Patienten - möglichst zum Zeitpunkt der Diagnose und kontinuierlich im

Behandlungsverlauf.

Durch den Einsatz standardisierter Fragebögen zur Erhebung von psychosozialer Belastung sowie der direkten Fragestellung nach dem psychoonkologischen Unterstützungswunsch der Betroffenen wird eine allgemeine Grundlage für die Auswahl weiterer Unterstützungsangebote geschaffen [4]. Eine anerkannte und validierte Screeeningmethode ist bspw. das Distress-Thermometer [8].

Gibt bspw. bei stationärer Aufnahme ein Patient im Belastungsscreening einen erhöhten Wert an oder äußert er den Wunsch nach psychoonkologischer Unterstützung, erfolgt eine persönliche Kontaktaufnahme durch den Psychoonkologen. Ein erstes persönliches Gespräch wird als eine wichtige Grundlage für den Aufbau einer Vertrauensbasis und zur Reduktion von Hemmungen, psychosoziale Hilfe in Anspruch zu nehmen, betrachtet. Beispielsweise kann durch eine frühe ausführliche Beratung über mögliche Unterstützungsangebote (z. B. Krebsberatungsstellen, Selbsthilfegruppe) informiert werden, um es Betroffenen zu erleichtern, diese im Laufe der Behandlung aufzusuchen [9]. Ferner dient das psychodiagnostische Gespräch zur Einschätzung der psychischen Belastungssituation des Betroffenen und dessen möglichen Unterstützungsbedarf. An den Erstkontakt kann sich die Einleitung weiterer therapeutischer Maßnahmen anschließen, oder der Patient wird an einen niedergelassenen Kollegen weitervermittelt.

\section{Psychoonkologische Therapie}

Die zumeist eingesetzte Therapieform in der Psychoonkologie ist die ressourcenorientierte Kurzpsychotherapie. Diese richtet sich an die Förderung von Bewältigungsmöglichkeiten des Patienten durch die Aktivierung von Stärken und Ressourcen, die den Betroffenen innewohnen. Lösungsansätze können so erarbeitet werden, ohne einen problemorientierten Fokus bei den ohnehin stark belasteten Patienten zu verfolgen. Ferner können einfache psychologische Techniken wie Entspannungs-, Imaginations- oder Achtsamkeitsübungen vermittelt werden. Zusätzlich gibt es eine Reihe von Interventionen, die auf die spezifischen Probleme von Krebspatienten und ihren Angehörigen ausgerichtet sind, wie bspw. die Progredienzangst, partnerschaftliche Bewältigung von Krebserkran- kungen oder der Umgang mit existenziellen Themen wie Tod und Sterben.

\section{Wirksamkeit psychotherapeutischer und medikamentöser Interventionen}

Insgesamt belegen die Ergebnisse einer aktuellen Metaanalyse aus 198 randomisierten, kontrollierten Studien psychoonkologischer Interventionen, dass psychoonkologische Therapieansätze effektiv und evidenzbasiert wirksam sind $[10,11]$. Untersucht wurde der Effekt verschiedener psychoonkologischer Interventionen auf den emotionalen Distress, die Lebensqualität sowie depressive und ängstliche Symptome erwachsener Krebspatienten. Die Analysen zeigen, dass sowohl im Einzel- und Gruppensetting kleine bis mittlere Effekte erzielt werden konnten. Die Behandlungen wirkten sich positiv auf die wahrgenommene Lebensqualität der Betroffen aus und reduzierten ihren empfundenen emotionalen Distress sowie ihre depressiven und ängstlichen Symptome. Diese Effekte konnten sowohl direkt nach der Akutbehandlung als auch über eine Periode von einem halben Jahr und länger nach der Behandlung nachgewiesen werden, auch wenn diese insgesamt kleiner waren als bei vergleichbaren psychotherapeutischen Studien. Durch die Vorauswahl hoch belasteter Patienten konnten jedoch auch größere Effekte nachgewiesen werden. Des Weiteren wurde eine Dosis-Wirkungs-Beziehung ermittelt, die belegt, dass längere Interventionszeiträume dauerhaftere Erfolge hervorbrachten.

Ein ähnliches Ergebnis zeigt auch ein aktuelles Review über den Einsatz von Psychopharmaka, insbesondere Antidepressiva, bei Krebspatienten. Die Ergebnisse belegen, dass depressive Symptome während der akuten Behandlungsphase durch den Einsatz von Antidepressiva verringert werden konnten. Allerdings belegte jedoch keine der verwendeten Studien die Wirksamkeit der Behandlung über 12 Wochen hinaus. Es lässt sich keine allgemein gültige Empfehlung zur klinischen Anwendung aus den Ergebnissen ableiten und über jede antidepressive Therapie bei onkologischen Patienten muss individuell entschieden werden, mit besonderer Beachtung der speziellen Wechselwirkungen mit der onkologischen Behandlung, z. B. Chemotherapeutika.

\section{Wie sind psychoonkologische Unterstützungsangebote organisiert?}

Die psychoonkologische Versorgung wird als Stepped-Care-Modell, also als gestufte Versorgung organisiert. Psychoonkologie ist damit Aufgabe aller an der Behandlung beteiligter Professionen, von Pflegekräften und onkologisch behandelnden Ärzten bis zu Psychotherapeuten. Bei emotionaler Belastung kümmern sich zunächst der behandelnde Arzt oder die Krankenpflege; erst bei spezifischen Problemen wie erhöhte Ängstlichkeit oder Depressivität wird der Psychoonkologische Psychotherapeut tätig.

Das multiprofessionelle Team versucht so, die Unterstützung an den Schweregrad der Belastung und die individuellen Bedürfnisse der Patienten anzupassen, um auch die Ressourcen aller Beteiligten so effektiv wie möglich zu nutzen [12]. Im Idealfall besteht ein System, das Behandlungen liefert und diese kontinuierlich nach 
ihrer aktuellen Passung an die Bedürfnislage des Patienten überprüft, sodass die Betroffenen so gezielt wie möglich und gleichzeitig auch so ressourcenschonend wie möglich behandelt werden.

Beteiligt an diesem gestuften Unterstützungsangebot sind alle im deutschen Gesundheitssystem vorhanden Sektoren, im Wesentlichen sind dies der stationäre (und teilstationäre), der ambulante und der Reha-Sektor. Im ambulanten Sektor erfolgt die psychotherapeutische Versorgung von Krebspatienten im Rahmen der Richtlinienpsychotherapie (idealerweise haben die Psychotherapeuten eine PSO-Zusatzqualifikation) sowie in den Klinikambulanzen; die Beratung erfolgt hauptsächlich in den Krebsberatungsstellen.

\section{Herausforderungen und Perspektiven}

Eine große Herausforderung ist die Verbesserung der Koordination und Kooperation zwischen den Behandlern und den verschiedenen Behandlungssektoren, damit die Möglichkeiten der aufgezeigten multiprofessionellen und sektorübergreifenden Modelle ausgeschöpft werden können. Eine Lösung könnten integrierte Versorgungsmodelle sein, an deren Entwicklung aktuell bereits gearbeitet wird. Eine weitere Herausforderung ist die Verbesserung der bislang mangelhaften flächendeckenden Versorgung in ländlichen Regionen, da die onkologischen Zentren nahezu ausschließlich in Städten bestehen und die Patienten aus ländlichen Regionen kaum Zugang zu psychoonkologischen Unterstützungsangeboten haben [4]. Wachsendes Interesse gilt E-Health-Angeboten, die psychoonkologische Hilfen online über das Internet auch in schwerer zugänglichen Regionen verfügbar machen.

Auch werden trotz des hohen Bedarfs faktisch nur wenige Patienten mit einer Krebserkrankung in der ambulanten psychotherapeutischen Regelversorgung behandelt. Möglicherweise war das psychotherapeutische Angebot bisher nicht bedarfsgerecht organisiert, denn Krebspatienten benötigen i.d.R. kurzfristige und flexible Terminangebote und eine niederschwellige Erreichbarkeit der Praxis. Es bleibt abzuwarten, ob die neuen Psychotherapie-Richtlinien mit der verpflichtenden Einrichtung von Sprechstunden hier Abhilfe schaffen. Allerdings ist eine Psychotherapie immer an die Voraussetzung der Diagnose einer psychischen Störung gebunden. Ambulante Beratungsangebote werden von den Krankenkassen bisher nicht finanziert. Die bestehenden Krebsberatungsstellen, die ambulante Beratung anbieten, sehen sich deshalb bis jetzt mit einer schwierigen Finanzierungslage konfrontiert [12].
Interessenkonflikt

Die Autoren erklären, dass kein Interessenkonflikt besteht.

\section{Literatur}

[1] AWMF. Leitlinienprogramm Onkologie (Deutsche Krebsgesellschaft, Deutsche Krebshilfe, AWMF): S3 Leitlinie Psychoonkologische Diagnostik, Beratung und Behandlung von erwachsenen Krebspatienten, AWMF-Registernummer: 032/051OL https://www.awmf.org/ leitlinien/detail/II/032-051OL.html Zugegriffen: 12.12.2017

[2] Baumeister H, Hutter N, Bengel J, Härter M. Quality of life in medically ill persons with comorbide mental disorders: A systematic review and meta analysis. Psychother Psychosom 2011; 80: 275-286

[3] Tschuschke V. Psychoonkologie. Psychologische Aspekte der Entstehung und Bewältigung von Krebs. Psychotherapeut 2012; 57: 191-192

[4] Weis ], Giesler ]. Versorgungsforschung in der Psychoonkologie. Der Onkologe 2017; 23: 893-899

[5] Zeiss T, Reuter K. Tumortherapie-Psychoonkologie: Qualitätsmerkmal in der Krebstherapie. Pharma Fokus Online 2014; 1: 31-35

[6] HeckI U, Singer S, Wickert M, Weis ]. Aktuelle Versorgungsstrukturen in der Psychoonkologie. Nervenheilkunde 2011; 30: 124-130

[7] Herschbach P, Weis J. Screeningverfahren in der Psychoonkologie: Testinstrumente zur Identifikation betreuungsbedürftiger Krebspatienten, Eine Empfehlung der PSO für die psychoonkologische Behandlungspraxis. Berlin: Deutsche Krebsgesellschaft e.V.; 2010

[8] Mehnert A, Müller D, Lehmann C, Koch U. Die deutsche Version des NCCN Distress-Thermometers. Empirische Prüfung eines ScreeningInstruments zur Erfassung psychosozialer Belastung bei Krebspatienten. Z Psychiatrie. Psychologie und Psychotherapie 2006; 54: 213-223

[9] Dilworth S, Higgins I, Parker V et al. Patient and health professional's perceived barriers to the delivery of psychosocial care to adults with cancer: a systematic review. Psychooncology 2014; 23: 601-612

[10] Faller $\mathrm{H}$, Schuler M, Richard M et al. Effects of psychooncological interventions on emotional distress and quality of life in adult cancer patients: systematic review and meta-analysis. J Clin Oncol 2013; 31: 782-793

[11] Ostuzzi G, Matcham F, Dauchy S et al. Antidepressants for the treatment of depression in people with cancer. Cochrane Database Syst Rev 2015; CD011006

[12] Weis J, Giesler J. Standards in der psychosozialen Versorgung von Krebspatienten. Der Onkologe 2016; 22: 198-205 